\section{A Coordinated Control Strategy of Voltage Regulation in Power System based on Multi-Agent System}

Mohamed Shawky El Moursi ${ }^{*}$ and Hasan Mehrjerdi

IEEE, Masdar Institute of Science and Technology, Abu Dhabi, UAE

"Correspondingauthor: Mohamed Shawky El Moursi, IEEE, Masdar Institute of Science and Technology, Abu Dhabi, UAE, Tel: +971501232070; E-mail: melmoursi@masdar.ac.ae

Rec date: Feb18, 2014; Acc date: Apr 16, 2014; Pub date: Apr 25, 2014

Copyright: (C) 2014 El MoursiMS, et al. This is an open-access article distributed under the terms of the Creative Commons Attribution License, which permits unrestricted use, distribution, and reproduction in any medium, provided the original author and source are credited.

\begin{abstract}
The development of power system from centralized to decentralized control provides a great opportunities for multi agent control. This paper presents a coordination algorithm for power systems based on concept of agents and multi agent systems. Agents provides the ability to execute better controllability on large scale systems including power systems. Here, multi agent systems is defined to regulate voltage of the power system. Tertiary and secondary voltage control that applying controllers to regulate the voltage in the power system are designed by agents. Voltage can be regulated using agents to support security of the power system and avoid fluctuation.
\end{abstract}

Keywords: Agent; Multi agent system; Voltage control

\section{Introduction}

Recently with problem of large scale systems, coordination control for multi agent systems (MAS) become an interesting topic of study. The big advantages of MAS are better efficiency, higher robustness and less communication compared to centralized algorithms. There is a lot of application for MAS in engineering for example in formation control [1], flocking [2], rendezvous [3], and power system [4].

The electric power system is complex and distributed in nature where the power source and the load are dispersed geographically. The increase in demand for electricity cause the electrical networks operate to their limits which resulting a great vulnerability to disturbance. Recent examples such as loss in North America in 2003, Italy in 2003, Greece in 2004 and in European countries November 4, 2006 are some parts of this vulnerability [5-8]. Although power industry spends a lot of money to protect a power system against different disturbances but large interconnected power systems throughout the world are frequently subjected to widespread outages/blackouts which interrupt millions of consumers and cost billions of dollars.

The voltage violation/instability is a major cause of outages/ blackouts. In EN50160 standard, the voltage magnitude variation on both Low Voltage (LV) and Medium Voltage (MV) networks is limited within $\pm 10 \%$ of the nominal voltage during $95 \%$ of the time of a week. In fact, this problem has been studied and analyzed in the past, however, the recent series of failures in the different countries shows that it is always a problem for researchers and network operators, especially as networks are much more in demand these days and they sometimes find themselves in exploitative conditions unplanned.

Transport networks possess long lines and are characterized by a great distance from production centers. It is generally difficult to maintain constant voltage when the demand varies in highly loaded networks. Without control, the voltage becomes lower than the permissible voltage. This is harmful because it causes loss of energy without considering the risk of disruptions and equipment breakdowns.
This motivates us to study and find efficient and secure voltage regulation in a power system by identifying the most appropriate controls. Security voltage refers to the ability of a power system to maintain stable voltages within the ranges prescribed for all bars of the system following a change of operating conditions. The voltage violation occurs when the network is no longer able to meet increased demand for active or reactive power. This can lead to the shutdown of a significant portion (or total) of the power system. The time of voltage violation may vary from seconds to tens of minutes.

Currently, voltage regulation systems are centralized and performed through a central computer that supervises all the generators productions and adjust optimally the voltage set points of these generators. This centralized regulation algorithm must know the whole network configuration and also it needs a high number of components to perform both data acquisition and communication system. However, for a large-scale power system, it may become difficult to perform a centralized control system. Lots of events and/or modifications may arise in a distribution network and the limits of a centralized control system may be reached quickly.

The interest of power system society is moving from traditional power system toward an intelligent and more reliable power system, some research has been performed to consider application of secondary voltage control and decentralized controllers in power systems [9-13] and coordination between the controllers [14-16].

\section{Agent and multi agent system}

Analysis of the possibilities and benefits of implementing MAS shows that it is a suitable technology for the complex and highly dynamic operation of the power system network. In the MAS hierarchical control structure each agent handles three functional aspects: management, coordination, and execution [17-20].

Therefore, in this paper, a decentralization of voltage regulation using MAS technique is proposed to perform the coordination with flexibility and reliability in electrical network. Each agent has a local multi-objective optimization function and controls the voltage at its bus and participates in voltage regulation of whole system. 
In order to explore the potential benefits of MAS to power engineering and voltage regulation, the basic concepts and approaches associated with MAS need to be understood. This leads us to a basic but essential definition of the agent and MAS conceptions.

Agent technology belongs to the area of computational intelligences, because they enable systems with a certain degree of intelligent behavior. Intelligence in this context mainly means less user interactions and automated adaptation to changing environments. An agent is a computational entity such as a software program or a zone in power system acting upon its environment. The environment is simply everything external to the agent. In order to be situated in an environment, at least part of the environment must be observable to, or alterable by, the agent. The environment may be physical (e.g., the power system), and therefore observable through sensors.

As an intelligent entity, an agent operates flexibly and rationally in a variety of environmental circumstances given its perceptual and effectual equipment. As an interacting entity, an agent can be affected in its activities by other agents.

The MAS consists of two agents or more that cooperate with each other and coordinate their knowledge and activities to accomplish a common goal. It is important to recognize that there is no overall system goal, simply the local goals of each separate agent.

A key pattern of interaction in MAS is goal and task oriented coordination, both in cooperative and in competitive situations. In the case of cooperation several agents try to combine their efforts to accomplish as a group what the individuals cannot, and in the case of competition several agents try to get what only some of them can have.

MAS is a team of problem solving agents which can react intelligently and flexibly to changing operating conditions and demands from the surrounding processes. Intelligent talents, such as learning from experience, planning of actions or detection and identification of errors, used to be part of the process operator duties, are integrated into the MAS. The advantages are reflected in the avoidance of human operating errors, the enhancement of the reaction rate and performance, and the reduction of the operator's effort.

The paper is organized as follows: Section II describes the application of MAS in power systems and the conclusion is in Section IIII.

\section{Application of MAS in Power Systems}

MAS have different application in power system including:

Monitoring and Diagnostics

Distributed Control

Modeling and Simulation

Protection

The current approach of using a central SCADA system and several smaller distributed SCADA systems is no longer sufficient for certain control operations. An approach that provides intelligent, fast, and adaptable local control and decision making is required.

Applications currently being investigated in the field of power control includes:

Power system restoration;

Active distribution networks operation;
Microgrid control;

Control of ship board electrical systems.

A number of researchers are considering agent based approaches as an alternative to centralized power system management and control. Local decision-making would require agents capable of a range of actions, such as monitoring local conditions, controlling switchgear and other plant, and coordinating with other regions of the network.

The now existing network is too passive, not intelligent enough, and not able to control the different situations and is therefore vulnerable. The limitation imposed by the conventional fixed-structure controllers necessitates finding a novel control architecture that provides a controller with coordinated operation, adaptive action, self-learning ability, and fast control.

The main objective of this paper is to define control system architecture for electrical power system based on intelligent agent technology to enhance the voltage regulation and stability of electric power distribution systems during faulty conditions and disturbances. Self-healing strategy also can be considered in power system. Application of MAS can be developed as:

To determine agent and multi-agent approach in power system and develop it to dividing network with the lowest possible interactions;

To design an autonomous voltage regulation using MAS platform and to enhance the transient stability problem;

To develop a fully efficient and flexible distributed coordination mechanism based on MAS for decentralized control and build up a new robust control framework;

To decompose of state estimation problem for large electric power system by using the multi-agent approach;

To eliminateany voltage violations in power system contingent operation. In condition of no agent manipulate the whole system and each agent has a model of only its local subsystem online information exchange helps to achieve coordination

Maintaining the voltage at any point in the network, in a narrow band compatible with the design of materials.

To this we must avoid:

Too high voltages that cause aging and destruction of equipment connected;

Too low voltages which cause overloads in the lines and disrupt the proper functioning of certain protections;

Optimal use of available transmission capacity. In particular, the reactive power must be produced near where it is consumed;

Preventing the risk of voltage collapse.

The selection of models and scenarios disturbed appropriate for the study of phenomena is very important. The interaction of the system load and equipment, protection of generator, excitation systems, transformers with load tap changers, shunt compensation and load shedding plays an important role in this process.

Main solutions for controlling voltage deviations in a electrical network are summarized as follows:

By the components such as on-load tap changer (OLTC) of the HV/MV transformer. 
Reactive power control with compensators or distributed generators (DG's).

Distributed control algorithms for electric power systems are the most feasible and desirable means of controlling voltage deviations, and stability. They are performed in order to formulate the best possible local subsystem controls as part of an intelligent control structure for the whole system. This goal is employing MAS which work in a decentralized control regime in such complicated systems. However, it requires the communication and cooperation, if found necessary, not among all the agents but only between closely related agents.

In MAS strategy in power system for the regulation of the regional voltage profile an individual power system voltage controller, such as an indicator, automatic voltage regulator (AVR), static var compensator (SVC), OLTC transformers or a static synchronous compensator (STATCOM), will be represented by agents (software) that can operate autonomously with local targets or cooperate with other agents to achieve area tasks. Task sharing in the MAS can be achieved by either communication or local estimation. A superior agent is installed as a moderator to manage autonomous actions as well as to communicate with other agents.

In the power system, there could be many MAS's formed and each voltage controller could be the agent in more than one MAS. The conflict of the actions taken by any individual agent for the task sharing of different MAS's which it joins is avoided. Voltage regulation/stability can be divided as follows:

Breakdown of electricity networks into subnets and determination of agents;

Determination of interactions between agents;

Setting automatic voltage regulation for agents;

Setting coordinated among the agents;

Using Contract Net Protocol (CNP) as a popular mechanism for negotiation.

When a voltage violation occurs at a bus, the corresponding agent of that bus detects the problem and sends an information message to its moderator. The moderator sends a Call for Proposal (CFP) message to every agent within its vicinity. The content of the message includes the desired voltage change at control bus.

Based on the power flow equations and the Jacobean matrix, the relationships between a bus voltage change and the bus power (active and reactive) injection are defined as so called sensitivity factors. The proposed method uses these factors to activate the autonomous characteristic of each controllable component to control a bus voltage. Bus power injections are managed to give an optimal voltage profile for the agent in the MAS.

Figure 1 show the general block diagram of MAS application in voltage regulation for power systems. As can be seen in this figure, agents are used for different part of voltage control algorithm.

By using the embedded functions in the Matlab/Simulink environment for modeling agents, a MAS platform can be created to exchange information among buses in the test network. Each bus agent implements its own voltage control algorithm with interactions from its agent neighbors.

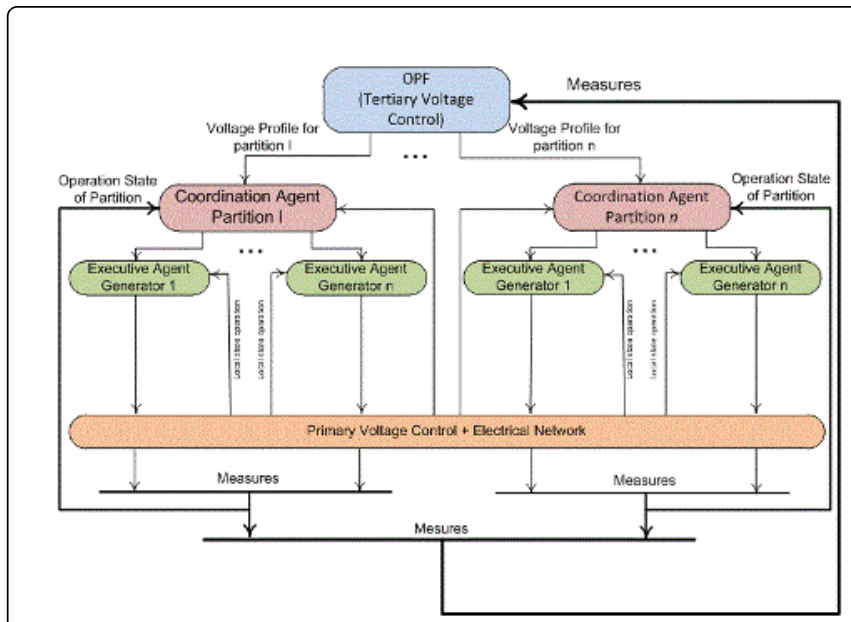

Figure 1: Voltage Control based on MAS in power systems

\section{Conclusion}

MAS has different applications in engineering and power systems. Applications of MAS in power system including monitoring, control, modelling, protection and so on. The proposed methodology of voltage control by MAS has the advantage of being relatively secure, simple and reliable. MAS is used for coordination of voltage in different layers of control including primary, secondary and tertiary. Controllers have been defined to avoid voltage violation using MAS.

\section{References}

1. Park BS, Park JB, Choi YH (2011) "Adaptive formation control of electrically driven nonholonomic mobile robots with limited information," IEEE Trans. Syst Man Cybern B Cybern 41: 1061-1075.

2. Olfati-Saber R (2006) "Flocking for multiagent dynamic systems: Algorithms and theory," IEEE Trans. Automat Control 51: 401-420.

3. Dimarogonas DV, Kyriakopoulos KJ (2007) "On the rendezvous problem for multiple nonholonomic agents," IEEE Trans. Automat Control 52: 916-922.

4. Sheng G, Xiuchen J, Dapeng D, Guangyu T (2009) "Framework and implementation of secondary voltage regulation strategy based on multiagent technology," International Journal of Electrical Power and Energy Systems 31:67-77.

5. Andersson G, Donalek P, Farmer R (2005) "Causes of the 2003 Major Grid Blackouts in NorthAmerica and Europe, and Recommended Means to Improve System Dynamic Performance," IEEE Transactions on Power Systems 20: 1922-1928.

6. Corsi S, Sabelli C (2004) "General Blackout in Italy Sunday September 28 2003, h.03:28:00," IEEE Power Engineering Society General Meeting 2: 1691-1702.

7. Vournas CD, Nikolaidis VC, Tassoulis A (2005) "Experience from the Athens Blackout of July 12, 2004," IEEE Power Tech.

8. Chen X, Deng C, Chen Y, Li C (2007) 'Blackout prevention: Anatomy of the blackout in Europe', International Power Engineering Conference, IPEC 2007: 928-932.

9. Mehrjerdi H, Lefebvre S, Saad M, Asber D (2013) "A Decentralized Control of Partitioned Power Networks for Voltage Regulation and Prevention against Disturbance Propagation," IEEE Trans. Power Syst 28: 1461-1469.

10. Mehrjerdi H, Ghahremani E, Lefebvre S, Saad M, Asber D (2013) "An Authenticated Voltage Control of Partitioned Power Networks with 
Citation: Mehrjerdi H, Moursi MSE (2014) A Coordinated Control Strategy of Voltage Regulation in Power System based on Multi-Agent System. Ind Eng Manage 3: 128. doi:10.4172/2169-0316.1000128

Page 4 of 4

Optimal Allocation of STATCOM Using Heuristic Algorithm," IET Gener Transm Distrib 7: 1037-1045.

11. El-Moursi MS (2010) A novel line drop secondary voltage control algorithm for variable speed wind turbines. Wind Energy 13: 633-655.

12. El-Moursi MS, Joos G (2009) Optimal Tracking Secondary Voltage Control for the DFIG Wind Turbines and compensator devices. Electric Power Systems Research 79: 1705-1716.

13. El-Moursi MS, Joos G, Abbey C (2008) A Secondary Voltage Control Strategies for Transmission Level Interconnection of Wind Generation. IEEE Transactions on power Electronics 23: 1178-1190.

14. Mehrjerdi H, Lefebvre S, Saad M, Asber D (2013) Coordinated Control Strategy ConsideringEffect of Neighborhood Compensation for Voltage Improvement in Transmission Systems. IEEE Trans Power Syst 28: 4507-4515.

15. El Moursi MS, Zeineldin HH, Kirtley JL, Alobeidli K (2014) A Dynamic Master/Slave Reactive Power-Management Scheme for Smart Grids With Distributed Generation. IEEE Transactions on Power Delivery, In press, 2014.
16. El-Moursi MS, Mansour HAR, Birgitte BJ (2011) Coordinated Voltage Control Scheme for SEIG-Based Wind Park Utilizing Substation STATCOM and ULTC Transformer. IEEE Transactions on Sustainable Energy 2: 246-255.

17. Nguyen PH, Myrzik JMA, Kling WL (2008) Coordination of Voltage Regulation in Active Networks. IEEE: 1-6.

18. Wang HF (2001) Multi-agent coordination for the secondary voltage control in power-system contingencies. IEE Proceedings on Generation, Transmission and Distribution 148: 61-66.

19. McArthur SDJ, Davidson EM, Catterson VM (2007) Multi-Agent Systems for Power Engineering Applications-Part II: Technologies, Standards, and Tools for Building Multi-agent Systems. IEEE Transactions on Power Systems 22: 1743-1752.

20. McArthur SDJ, Davidson EM, Catterson VM (2007) Multi-Agent Systems for Power Engineering Applications-Part II: Technologies, Standards, and Tools for Building Multi-agent Systems. IEEE Transactions on Power Systems 22: 1753-1759. 